\title{
PEAK POWER MINIMIZATION IN SYMBOL-LEVEL PRECODING FOR COGNITIVE MISO DOWNLINK CHANNELS
}

\author{
Maha Alodeh Danilo Spano Symeon Chatzinotas and Björn Ottersten \\ Interdisciplinary Centre for Security Reliability and Trust (SnT), University of Luxembourg. \\ E-mails: $\{$ maha.alodeh, danilo.spano, symeon.chatzinotas, bjorn.ottersten@uni.lu\}
}

\begin{abstract}
This paper proposes a new symbol-level precoding scheme at the cognitive transmitter that jointly utilizes the data and channel information to reduce the effect of nonlinear amplifiers, by reducing the maximum antenna power under quality of service constraint at the cognitive receivers. In practice, each transmit antenna has a separate amplifier with individual characteristics. In the proposed approach, the precoding design is optimized in order to control the instantaneous power transmitted by the antennas, and more specifically to limit the power peaks, while guaranteeing some specific target signal-to-noise ratios at the receivers and respecting the interference temperature constraint imposed by the primary system. Numerical results show the effectiveness of the proposed scheme, which outperforms the existing state of the art techniques in terms of reduction of the power peaks.
\end{abstract}

\section{INTRODUCTION}

The paradigm of cognitive radios has been proposed as a promising technology that can change the way we look at the spectrum [1][3]. The key idea of their implementation is to allow opportunistic transmissions to share the wireless media. Thus, two initial hierarchal levels have been defined: primary level and secondary level (the users within each level are called primary users (PU) and cognitive users (CU) respectively). The interaction between these two levels is determined by the agility of the secondary level and the predefined constraints imposed by the primary level [3]. Overlay, underlay and interweave are three general implementations which regulate the coexistence terms of the both systems. The first two implementations allow simultaneous transmissions, which leads to better spectrum utilization in comparison to the last one, which allocates the spectrum to the secondary system by detecting the absence of the primary one [4].

The form of integration in this work is defined by cooperation between the two levels in the cognitive interference channel. The cooperation can aid the primary network to satisfy the quality of service (QoS) or enhance the rate of its own users by backhauling its data through the secondary [5]- [8]; Cognitive base station (CBS) can operate as relays for primary messages and as regular base stations to serve their secondary users. The secondary system benefits by providing a service to its users. This kind of cognitive implementation fits with practical overlay cognitive definition, as the PU is being served from both the primary base station (PBS) and the CBSs

This work is supported by Fond National de la Recherche Luxembourg (FNR) project, Spectrum Management and Interference Mitigation in Cognitive Radio Satellite Networks (SeMiGod), SATellite SEnsor NeTworks for spectrum monitoring (SATSENT), and H2020 project Shared Access Terrestrial-Satellite Backhaul Network enabled by Smart Antennas (SANSA) by performing relaying between them to make primary data accessible by the CBS. Sometimes the primary symbols cannot be available to the secondary system, as a result the secondary system needs to take the sufficient precautions to protect the primary system from the interference created by its own transmissions. It should be noted that the CBSs should be equipped with multiple antennas to handle multiuser transmissions, and to enable interference mitigation.

Interference in multiuser multiple-antenna system is a harmful factor that degrades the performance of wireless operators. Symbollevel precoding is a new paradigm that changes the interference into a favorable factor that can be exploited if we tackle the transmitted data frame at symbol level. At symbol level, the interference can be classified into: constructive and destructive ones. This classification is initially proposed in [12]; instead of fully inverting the channel to grant zero interference among the spatial streams, the proposed precoding suggests keeping the constructive interference while removing the destructive part by partial channel inversion. This technique is proven to outperform the traditional zero forcing precoding. A more advanced technique is proposed in [13], where an interference rotation is examined to make the interference constructive for all users. Moreover, a modified maximum ratio transmissions technique that performs unitary rotations to create constructive interference among the interfering multiuser streams is proposed [15]. Furthermore, a connection between symbol based constructive interference precoding and PHY multicast is established in [15]- [16]. The symbol-level precoding has been utilized in different applications such as cognitive radio [17] and physical-layer security [22]- [23]. In [17], we shape the interference between the cognitive users to have constructive characteristics without inducing any harmful impact on the primary receivers.

On the other hand, the nonlinearity of high-power amplifiers (HPAs) has a crucial effect on the performance of multiple antenna systems. In general, the HPA is assumed to operate in its linear region to ensure that the characteristics of the output symbols are not affected by the power amplification process, which is not necessarily the case in practical situations, particularly when the HPA operates at the medium and high-power signal levels. The non-linearities of HPA has influential impact at high input power, this results in nonuniform power amplification of each antenna response. As a consequence, the precoded waveforms suffer from distortions and thus they show bad performance [24]. In this work, we propose a symbollevel precoding scheme that controls the instantaneous per-antenna transmit power, thus minimizing the power peaks, which are detrimental with respect to the aforementioned non-linearity problem. In contrary to symbol-level precoding, the conventional schemes designs the precoding for an entire codeword, therefore, the transmitted power can be controlled only in average and not symbol by symbol [26]- [28]. In this paper, we want to limit the effects of non-linearities in the downlink of multiple-antenna cognitive sys- 
tem, while satisfying the quality of service demands of the cognitive users without violating interference temperature constraints imposed by primary system.

Notation: We use boldface upper and lower case letters for matrices and column vectors, respectively. $(\cdot)^{H},(\cdot)^{*}$ stand for Hermitian transpose and conjugate of $(\cdot) . \mathbb{E}(\cdot),\|\cdot\|$ and $\|\cdot\|_{\infty}$ denote the statistical expectation, the second norm and the infinite norm respectively. $\angle(\cdot),|\cdot|$ are the angle and magnitude of $(\cdot)$ respectively. $R(\cdot), \mathcal{I}(\cdot)$ are the real and the imaginary parts of $(\cdot)$.

\section{SYSTEM AND SIGNALS MODEL}

\subsection{System model}

We consider a cognitive radio network which shares the spectrum resource with a primary network. The primary network consists of a primary base station (PBS) that transmits signals to $K_{p}$ multiple primary users. The secondary cognitive network has a single cognitive base station (CBS), equipped with $\mathrm{M}$ antennas, serving $\mathrm{K}$ secondary users. Throughout this paper, we consider that and that each primary user and cognitive user is equipped with a single antenna. Due to the sharing of the same frequency band, the received signal at the primary user is interfered by the signals transmitted from CBS. Similarly, the received signals at the secondary users are interfered by the signal transmitted from the PBS.

\subsection{Signal model}

Assume that in one time slot, a block of information symbols $\mathbf{d}=$ $\left[d_{1}, d_{2}, \ldots, d_{K}\right]^{T}$ are sent from the CBS in which $d_{k}, k=1, \ldots, K$ is the desired signal for user $k$. With a proper beamforming (which will be specified later), the transmit signal is given by

$$
\mathbf{x}=\mathbf{W d}
$$

where $\mathbf{W}=\left[\mathbf{w}_{1}, \mathbf{w}_{2}, \ldots, \mathbf{w}_{K}\right]$ denotes the transmit beamforming matrix for the secondary system while $\mathbf{w}_{k} \in \mathbb{C}^{M \times 1}$ denotes the beamforming vector for $k^{t h}$ secondary user. The received signal at the $k^{\text {th }}$ cognitive user is given by:

$y_{s, k}=\mathbf{h}_{s, k} \mathbf{w}_{k} d_{k}+\sum_{j \in K, j \neq k} \mathbf{h}_{s k} \mathbf{w}_{j} d_{j}+\sum_{i=1}^{K_{p}} \mathbf{h}_{s p, k} \mathbf{g}_{i} d_{p, i}+n_{k}$,

and the received signal at PU's receiver is given by

$$
y_{p, k}=\mathbf{h}_{p, k} \mathbf{g}_{k} d_{p, k}+\mathbf{h}_{p, k} \sum_{\substack{\forall i \in K_{p}, i \neq k \\ \text { interference from other PUs }}} \mathbf{g}_{i} d_{p, i}+\mathbf{h}_{p s, k} \sum_{\substack{j \in K \\ \text { interference from CUs }}} \mathbf{w}_{j} d_{j}+n_{p, k}
$$

where $\mathbf{h}_{s k} \in \mathbb{C}^{1 \times M}$ and $\mathbf{h}_{s p, k} \in \mathbb{C}^{1 \times N_{p}}$ are the channels between the CBS and the PBS respectively and the $k^{t h}$ cognitive user. While $\mathbf{h}_{p}$ and $\mathbf{h}_{p s}$ denote the channel between the PBS and PU, CBS and PU respectively. $\mathbf{g}_{i} \in \mathbb{C}^{N_{p} \times 1}, d_{p, i}$ denote the beamforming vector used by the PBS to serve its $i^{\text {th }}$ primary users and the transmitted symbol from the PBS to $k^{t h}$ primary user. $n_{k}$ and $n_{p, k}$ are additive i.i.d. complex Gaussian noise with zero mean and variance $\sigma_{k}^{2}$ at the $k^{\text {th }} \mathrm{CU}$ and PU respectively. The channel state information from the CBS to CUs and from CBS to PUs are assumed to be known perfectly at CBS.

\section{PEAK POWER MINIMIZATION SYMBOL-LEVEL PRECODING FOR THE COGNITIVE DOWNLINK}

In this section, we design a symbol-level precoding technique at the cognitive base station to reduce the peak spatial power to limit the effect of HPA nonlinearities.

\subsection{Relaxed Interference Constraint}

The precoding aims at exploiting the constructive interference among the cognitive users without violating the interference temperature constraint imposed by the primary system $\mathcal{I}_{t h}$. The optimization can be formulated as

$$
\begin{aligned}
& \mathbf{w}_{1}, \ldots, \mathbf{w}_{K}= \arg \min _{\mathbf{w}_{1}, \ldots, \mathbf{w}_{K}} \max _{i}\left[\sum_{k=1}^{K} \mathbf{w}_{k} d_{k} \sum_{k=1}^{K} d_{k}^{H} \mathbf{w}_{k}^{H}\right]_{i, i=1}^{M} \\
& \text { s.t. } \quad\left\{\begin{array}{l}
\mathcal{C} 1: \angle\left(\mathbf{h}_{s, j} \sum_{k=1}^{K} \mathbf{w}_{k} d_{k}\right)=\angle\left(d_{j}\right), \forall j \in K \\
\mathcal{C} 2: \frac{\left\|\mathbf{h}_{s, j} \sum_{k=1}^{K} \mathbf{w}_{k} d_{k}\right\|^{2}}{\sigma^{2}+\left\|\mathbf{h}_{s p, j} \sum_{i=1}^{K} \mathbf{g}_{i}\right\|^{2}} \unlhd \kappa_{j}^{2} \zeta_{j} \\
\mathcal{C}_{3}:\left\|\mathbf{h}_{p s, n} \sum_{k=1} \mathbf{w}_{k} d_{k}\right\|^{2} \leq \mathcal{I}_{t h}, \forall n \in K
\end{array}, \forall j\right)
\end{aligned}
$$

where $\unlhd$ indicates that the symbols should locate in the correct detection region, $\zeta_{j}$ is the SNR target (which is associated with the modulation order) and $\kappa_{j}$ denotes the short-term factor $\kappa_{j}=\left|d_{j}\right| / \sqrt{\mathbb{E}_{\mathcal{D}}\left[\left|d_{j}\right|^{2}\right]}$ changes on a symbol-basis and adjusts the long-term SINR based on the amplitude of the desired symbol. The first two sets of constraints $\mathcal{C}_{1}$ and $\mathcal{C}_{2}$ grant the reception of the data symbols with certain signal to noise ratio (SNR) level. The third constraint $\mathcal{C}_{3}$ is to protect the PU from the secondary systems transmissions. In order to solve (4), we formulate it by using $\mathbf{x}=\sum_{k=1}^{K} \mathbf{w}_{k} d_{k}$ as the following

$$
\begin{aligned}
\mathbf{x}= & \arg \operatorname{minmax}_{\mathbf{x}}\left|x_{i}\right|^{2} \\
\text { s.t. } & \begin{cases}\mathcal{C} 1: \frac{\mathbf{h}_{s, j} \mathbf{x}+\mathbf{x}^{H} \mathbf{h}_{s, j}^{H}}{2} \unlhd \kappa_{j} \sqrt{\psi_{j} \zeta_{j}} \mathcal{R}\left\{d_{j}\right\} \quad, \forall j \in K \\
\mathcal{C} 2: \frac{\mathbf{h}_{s, j} \mathbf{x}-\mathbf{x}^{H} \mathbf{h}_{s, j}^{H}}{2 i} \unlhd \kappa_{j} \sqrt{\psi_{j} \zeta_{j}} \mathcal{I}\left\{d_{j}\right\} & , \forall j \in K \\
\mathcal{C}_{3}:\left\|\mathbf{h}_{p s, n} \mathbf{x}\right\|^{2} \leq \mathcal{I}_{t h} & , \forall n \in K_{p} .\end{cases}
\end{aligned}
$$

where $\psi_{j}=\sigma^{2}+\left\|\mathbf{h}_{s p, j} \sum_{i}^{K_{p}} \mathbf{g}_{i}\right\|^{2}$. In this work, we focus on the multi-level modulations ${ }^{1}$. In 16-QAM, for the outermost symbols in the first quadrant $\unlhd$ means $\geq$ in real and imaginary and the innermost $\unlhd$ means $=^{2}$. The previous optimization problem can be reformulated as infinite norm minimization one as:

(3) $\mathbf{x}=\arg \min _{\mathbf{x}}\|\mathbf{x}\|_{\infty}^{2}$

$$
\text { s.t. } \begin{cases}\mathcal{C} 1: \frac{\mathbf{h}_{s, j} \mathbf{x}+\mathbf{x}^{H} \mathbf{h}_{s, j}^{H}}{2} \unlhd \kappa_{j} \sqrt{\psi_{j} \zeta_{j}} \mathcal{R}\left\{d_{j}\right\} \quad, \forall j \in K \\ \mathcal{C} 2: \frac{\mathbf{h}_{s, j} \mathbf{x}-\mathbf{x}^{H} \mathbf{h}_{s, j}^{H}}{2 i} \unlhd \kappa_{j} \sqrt{\psi_{j} \zeta_{j}} \mathcal{I}\left\{d_{j}\right\} \\ \mathcal{C}_{3}:\left\|\mathbf{h}_{p s, n} \mathbf{x}\right\| \leq \sqrt{\mathcal{I}_{t h}} \quad, \forall n \in N .\end{cases}
$$

where $\mathcal{C}_{1}, \mathcal{C}_{2}$ are affine constraints, and $\mathcal{C}_{3}$ is a convex constraint. This problem can be solved optimally using second order cone programing [29]. Without interference temperature constraint $\mathcal{C}_{3}$, the problem can be solved using linear programming [29].

\footnotetext{
${ }^{1}$ For PSK modulation, it has been discussed thoroughly in [17].

${ }^{2}$ For more details, please look at eq.(13) -(16).
} 


\subsubsection{Zero Interference Constraint}

If the PU cannot tolerate any interference, the secondary transmissions should be in the null space of the channel between CBS and PU. To guarantee zero interference is to project the transmitted signal $\mathrm{x}$ in the null space of the channel matrix between CBS transmitter and the remaining receivers. The optimization in (7) can be reformulated as:

$$
\begin{aligned}
& \mathbf{x}=\arg \min _{\mathbf{x}}\|\mathbf{x}\|_{\infty}^{2} \\
& \text { s.t. } \begin{cases}\mathcal{C} 1: \frac{\mathbf{h}_{s, j} \mathbf{x}+\mathbf{x}^{H} \mathbf{h}_{s, j}^{H}}{2} \unlhd \kappa_{j} \sqrt{\psi_{j} \zeta_{j}} \mathcal{R}\left\{d_{j}\right\} \quad, \forall j \in K \\
\mathcal{C} 2: \frac{\mathbf{h}_{s, j} \mathbf{x}-\mathbf{x}^{H} \mathbf{h}_{s, j}^{H}}{2 i} \unlhd \kappa_{j} \sqrt{\psi_{j} \zeta_{j}} \mathcal{I}\left\{d_{j}\right\} \\
\mathcal{C}_{3}: \mathbf{h}_{p s, n} \mathbf{x}=0, \forall j \in K\end{cases}
\end{aligned}
$$

Also, this optimization can be solved using linear programming [29].

\section{SPECIAL CASE: SYMBOL-LEVEL PRECODING FOR ONE DIMENSIONAL MODULATION}

For one dimensional modulations, it is possible to exploit the complex dimension of the channels to serve more users than the number of transmit antennas [25]. Assuming that the adopted modulation is BPSK, the optimization problem can be formulated as:

$$
\begin{aligned}
\mathbf{x}= & \arg \min _{\mathbf{x}}\|\mathbf{x}\|_{\infty} \\
\text { s.t. } & \left\{\begin{array}{l}
\mathcal{C} 1: \frac{\mathbf{h}_{s, j} \mathbf{x}+\mathbf{x}^{H} \mathbf{h}_{s, j}^{H}}{2} \unlhd \sqrt{\psi_{i} \zeta_{j}} \mathcal{R}\left\{d_{j}\right\} \quad, \forall j \in K \\
\mathcal{C}_{2}:\left\|\mathbf{h}_{p s, n} \mathbf{x}\right\| \leq \sqrt{\mathcal{I}_{t h}}, \forall n \in K_{p} .
\end{array}\right.
\end{aligned}
$$

Since single dimension is exploited in BPSK modulation, the optimization in (8) has $K$ less constraints than (7). The other dimension can be used to serve $K$ additional user as long as they adopt BPSK modulation. For the case of users have different rate demands, which can be translated into different allocated modulations, we can serve additional user if one user allocated BPSK modulation.

\section{NUMERICAL RESULTS}

In order to assess the performance of the proposed transmissions schemes, Monte-Carlo simulations of the different algorithms have been conducted to study the performance of the proposed techniques and compare to the state of the art techniques. For the adopted channel, we assume that there is a large line of sight path (specular path) of known magnitude and there are also a strong number of independent paths, which can be modeled as:

$$
\begin{aligned}
& \mathbf{h}_{s, j}=\sqrt{\frac{\rho}{\rho+1}} \sigma \mathbf{a}\left(\theta_{j}\right)+\sqrt{\frac{1}{\rho}} \mathcal{C N}\left(\mathbf{0}_{1 \times M}, \sigma^{2} \mathbf{1}_{1 \times M}\right) \\
& \text { where } \\
& \mathbf{a}\left(\theta_{j}\right)=\frac{1}{\sqrt{M}}\left[1, e^{j m d \sin \theta_{j}}, \ldots, e^{j(M-1) m d \sin \theta_{j}}\right]
\end{aligned}
$$

and $m=\frac{2 \pi}{\lambda}, d$ is the inter-element spacing, $M$ is the number of uniform linear array (ULA)s elements, $\mathbf{0}_{1 \times M}$ and $\mathbf{1}_{1 \times M}$ are the vectors of all zeros and all ones respectively. The first term is the specular path arriving with uniform phase $\theta_{j}$ and the second one is due to the aggregation of the large number of reflected and scattered paths, idepedent of $\theta_{i} j$. The parameter $\rho$ is theso-called $\mathrm{k}$-factor and it is the ratio of the energy in the specular path to the energy in the scattered paths; the larger $\rho$ is, the more deterministic the channel becomes. The parameter $\sigma$ denotes the total energy received from all the paths.

\begin{tabular}{|l|l|l|}
\hline Acronym & Technique & equation \\
\hline CCIPM & $\begin{array}{l}\text { Cognitive Constructive Interference- } \\
\text { Power Minimization }\end{array}$ & $\begin{array}{l}(11), \\
{[17]}\end{array}$ \\
\hline CCIMPM & $\begin{array}{l}\text { Cognitive Constructive Interference } \\
\text { Maximum Power Minimization }\end{array}$ & $(7)$ \\
\hline ASP & Average Spatial Power & \\
\hline MSP & Maximum Spatial Power & \\
\hline
\end{tabular}

Table 1. Summary of the proposed, state-of-the-art algorithms, their related acronyms, and their related equations and algorithms

The magnitude of such a variable is said to follow the so-called Rician Distribution (9). The list of used acronyms in this section is summarized in Table 1.

The proposed techniques is compared to the state-of-art technique CCIPM [17], which can be mathematically formulated as the solution of the following optimization problem:

$$
\begin{aligned}
\mathbf{x}= & \arg \min _{\mathbf{x}}\|\mathbf{x}\|^{2} \\
\text { s.t. } & \left\{\begin{array}{l}
\mathcal{C} 1: \frac{\mathbf{h}_{s, j} \mathbf{x}+\mathbf{x}^{H} \mathbf{h}_{s, j}^{H}}{2} \unlhd \kappa_{j} \sqrt{\psi_{i} \zeta_{j}} \mathcal{R}\left\{d_{j}\right\} \quad, \forall j \in K \\
\mathcal{C} 2: \frac{\mathbf{h}_{s, j} \mathbf{x}-\mathbf{x}^{H} \mathbf{h}_{s, j}^{H}}{2 i} \unlhd \kappa_{j} \sqrt{\psi_{i} \zeta_{j}} \mathcal{I}\left\{d_{j}\right\} \\
\mathcal{C}_{3}:\left\|\mathbf{h}_{p s, n} \mathbf{x}\right\| \leq \sqrt{\mathcal{I}_{t h}}, \forall n \in K
\end{array}, \forall j \in K_{p} .\right.
\end{aligned}
$$

If we denote the real and imaginary part of the signal as follows

$$
\mathcal{I}_{j}=\frac{\mathbf{h}_{s, j} \mathbf{x}+\mathbf{x}^{H} \mathbf{h}_{s, j}^{H}}{2}, \mathcal{Q}_{j}=\frac{\mathbf{h}_{s, j} \mathbf{x}-\mathbf{x}^{H} \mathbf{h}_{s, j}^{H}}{2 i},
$$

the constraints $\mathcal{C}_{1}, \mathcal{C}_{2}$ for 8-QAM symbols can be written in detail as:

$$
\begin{gathered}
\mathcal{C}_{1}=\left\{\begin{array}{l}
\mathcal{I}_{j}=\sigma \sqrt{\psi_{j} \frac{\zeta_{j}}{3}} \mathcal{R}\left\{d_{j}\right\}, d_{j}=\frac{ \pm 1 \pm i}{\sqrt{2}} \\
\mathcal{I}_{j} \geq \sigma \frac{\sqrt{\psi_{j} \zeta_{j}}}{\sqrt{3}} \mathcal{R}\left\{d_{j}\right\}, d_{j}=\frac{3+i}{\sqrt{2}}, \frac{3-i}{\sqrt{2}} \\
\mathcal{I}_{j} \leq \sigma \frac{\sqrt{\psi_{j} \zeta_{j}}}{\sqrt{3}} \mathcal{R}\left\{d_{j}\right\}, d_{j}=\frac{-3+i}{\sqrt{2}}, \frac{-3-i}{\sqrt{2}}
\end{array}\right. \\
\mathcal{C}_{2}=\left\{\begin{array}{l}
\mathcal{Q}_{j} \geq \sigma \sqrt{\psi_{j} \frac{\zeta_{j}}{3}} \mathcal{I}\left\{d_{j}\right\}, d_{j}=\frac{ \pm 1+i}{\sqrt{2}}, \frac{ \pm 3+i}{\sqrt{2}}, \\
\mathcal{Q}_{j} \leq \sigma \sqrt{\psi_{j} \frac{\zeta_{j}}{3}} \mathcal{I}\left\{d_{j}\right\}, d_{j}=\frac{ \pm 1-i}{\sqrt{2}}, \frac{ \pm 3-i}{\sqrt{2}},
\end{array}\right.
\end{gathered}
$$

and for the 16-QAM symbols, they can be expressed as:

$$
\begin{gathered}
\mathcal{C}_{1}=\left\{\begin{array}{l}
\mathcal{I}_{j}=\sigma \sqrt{\psi_{j} \frac{\zeta_{j}}{5}} \mathcal{R}\left\{d_{j}\right\}, d_{j}=\frac{ \pm 1+ \pm i}{\sqrt{2}}, \frac{ \pm 1+ \pm 3 i}{\sqrt{2}} \\
\mathcal{I}_{j} \geq \sigma \sqrt{\psi_{j} \frac{\zeta_{j}}{5}} \mathcal{R}\left\{d_{j}\right\}, d_{j}=\frac{3+i}{\sqrt{2}}, \frac{3-i}{\sqrt{2}}, \frac{3+3 i}{\sqrt{2}}, \frac{3-3 i}{\sqrt{2}} \\
\mathcal{I}_{j} \leq 2 \sigma \sqrt{\psi_{j} \frac{\zeta_{j}}{5}} \mathcal{R}\left\{d_{j}\right\}, d_{j}=\frac{-3+i}{\sqrt{2}}, \frac{-3-i}{\sqrt{2}}, \frac{-3+3 i}{\sqrt{2}}, \frac{-3-3 i}{\sqrt{2}}
\end{array}\right. \\
\mathcal{C}_{2}=\left\{\begin{array}{l}
\mathcal{Q}_{j}=\sigma \sqrt{\psi_{j} \frac{\zeta_{j}}{5}} \mathcal{I}\left\{d_{j}\right\}, d_{j}=\frac{ \pm 1+ \pm i}{\sqrt{2}}, \frac{ \pm 3+ \pm i}{\sqrt{2}} \\
\mathcal{Q}_{j} \geq \sigma \sqrt{\psi_{j} \frac{\zeta_{j}}{5}} \mathcal{I}\left\{d_{j}\right\}, d_{j}=\frac{ \pm 1+3 i}{\sqrt{2}}, \frac{ \pm 3+3 i}{\sqrt{2}} \\
\mathcal{Q}_{j} \leq \sigma \sqrt{\frac{\psi_{j} \zeta_{j}}{5}} \mathcal{I}\left\{d_{j}\right\}, d_{j}=\frac{ \pm 1-3 i}{\sqrt{2}}, \frac{ \pm 3-3 i}{\sqrt{2}}
\end{array}\right.
\end{gathered}
$$

Fig. 1 depicts the transmit power of CCIPM and CCIMPM with respect to target SNR. The two main assumptions are all cognitive users have the same SNR target and the same modulation and all 


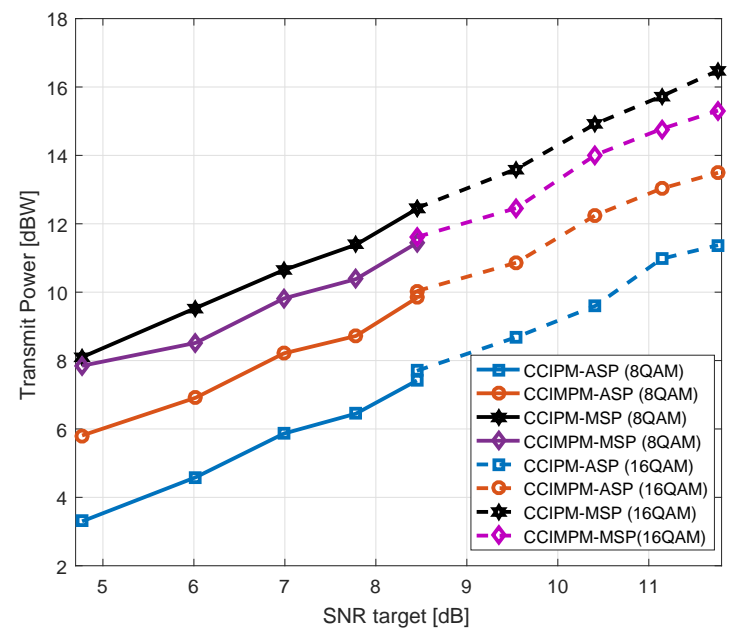

Fig. 1. Transmit Power vs SNR Target. Scenario: $N_{P U}=2$, $N_{C U}=10, M=12, \sigma_{h}^{2}=0 d B, \rho=2, \mathcal{I}_{t h}=0 d B$.

primary users have the same interference temperature constraints for the sake of simplicity. The adopted modulations can be adapted with respect to the SNR targets, we use 8-QAM and 16-QAM and it can be extended to any other multi-level modulation as long as the detection regions are specified appropriately [20]. In order to study the performance accurately, we use two metrics to compare the performance: the peak $\|\mathbf{x}\|_{\infty}^{2}$ and average $\|\mathbf{x}\|^{2} / M$ spatial power in order to capture the per-antenna performance. As expected, it can be noted that the required power for any case increases with the target SNR. In more details, CCIMPM attains better performance in terms of peak power with respect to the CCIPM approach which makes the per-antenna power more uniform. This comes at the expense of higher power consumption in comparison to CCIPM.

Fig. 2 illustrates the spatial peak power to average power ratio (SPAPR), which can be mathematically defined as $\frac{\|\mathbf{x}\|_{\infty}^{2}}{\|\mathbf{x}\|_{2}^{2}}$, with respect to system size. The performance of CCIPM and CCIMPM is studied to understand the trade-off between the peak and spatial average power. It can be noted that SPAPR of CCIPM increases with system size. In contrast to CCIPM, the SPAPR of CCIMPM decreases with the system size. This ensures that the per-antenna power is close to the average spatial power, which regulates the effect of HPA and limits amplitude distortions.

Fig. 3 depicts the transmit power with respect to the number of cognitive users, all of them adopt BPSK modulation. In the conventional precoding, the number of simultaneous cognitive users that the cognitive base station wants to serve and the number of primary users wants to protect should be equal to (or less than) the number of antennas at the cognitive base station. Since the symbol-level precoding exploits the constellation order, it can be noted that the number of simultaneous served used can be higher than the number of antennas. For example, if we assume that the cognitive base station has 12 antennas and the number of primary users equals to 2, the number of cognitive users can reach to 20 users receiving BPSK modulation.

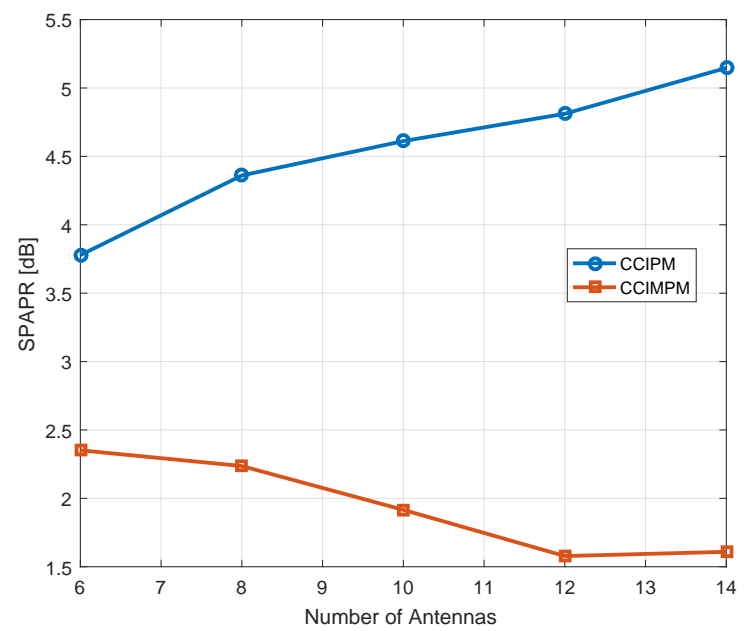

Fig. 2. Transmit Power vs Number of Cognitive Base Station Antennas $M$. Scenario: $N_{C U}=M-2, N_{P U}=2, \sigma_{h}^{2}=0 d B, \rho=2$, $\mathcal{I}_{t h}=0 d B$

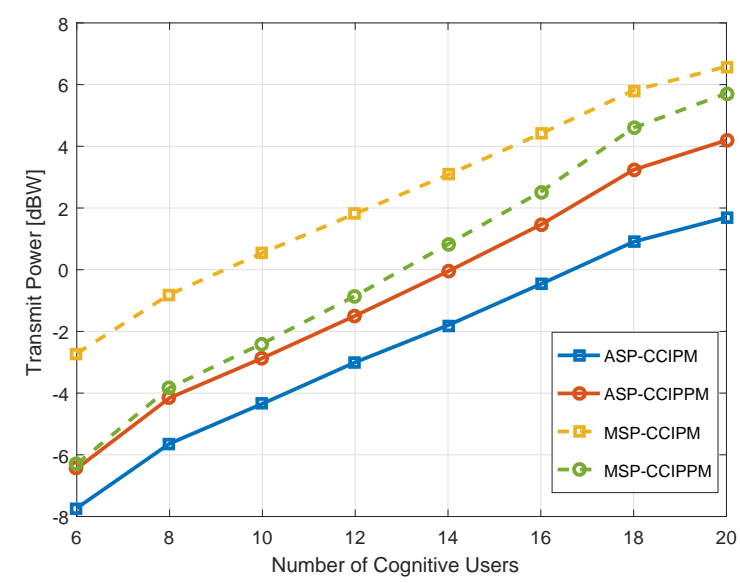

Fig. 3. Transmit Power vs Number of Cognitive users. Scenario: $M=12, N_{P U}=2, \sigma_{h}^{2}=0 d B, \rho=2, \mathcal{I}_{t h}=0 d B$

\section{CONCLUSIONS}

In this paper, we propose symbol-level precoding scheme for the downlink of cognitive underlay system. These techniques exploit the availability of channel information and data symbols to constructively correlate the transmission for cognitive users without violating the interference temperature at the primary users. This enables interference exploitation among the cognitive multiuser transmissions. The designed aims at minimizing the peak per-antenna power at CBS while guaranteeing a predefined received SNR at each cognitive users. Symbol-level precoding uses the signal domain, which is different from the conventional precoding, this enables the CBS to serve more users than the number of antennas using BPSK modulation. From the numerical results, it can be concluded that the proposed technique achieves higher spatial peak to average power ratio than the state-of-the art technique, which limits the effect of HPA in the system. 


\section{REFERENCES}

[1] Federal Communication Commission, Spectrum Policy Task Force, ET document no. 02-135, Nov. 2002.

[2] A. Goldsmith, S. A. Jafar, I. Maric, and S. Srinivasa, "Breaking spectrum gridlock with cognitive radios: An information theoretic perspective," IEEE, vol. 97, no. 5, pp. 894 - 914, May 2009.

[3] S. Haykin, "Cognitive Radio: Brain-Empowered Wireless Communications," IEEE Journal on Selected Areas in Communications, vol. 23, pp. 201-22, Feb. 2005.

[4] S. Srinivasa,S. A. Jafar, "Soft Sensing and Optimal Power Control for Cognitive Radio,"IEEE Transactions on Wireless Communications, vol. 9, no. 12, pp. 36383649, October 2010.

[5] J. Lv, R. Blasco-Serrano, E. Jorswieck, R. Thobaben and A. Kliks, "Optimal Beamforming in MISO Cognitive Channels with Degraded Message Sets," IEEE Conference on Wireless Communications and Networking (WCNC), April 2012.

[6] J. Lv, R. Blasco-Serrano, E. Jorswieck and R. Thobaben, "Linear Precoding in MISO Cognitive Channels with Causal Primary Message," IEEE International Symposium on Wireless Communications Systems (ISWCS), 2012.

[7] J. Lv, R. Blasco-Serrano, E. Jorswieck and R. Thobaben,"Multi-antenna transmission for underlay and overlay cognitive radio with explicit message-learning phase,"

[8] G. Zheng, S. H. Song, K. K. Wong, and B. Ottersten, "Cooperative Cognitive Networks: Optimal, Distributed and Low-Complexity Algorithms," IEEE Transaction on Signal Processing, vol. 61, no.11 , pp. 2778 - 2790, June 2013.

[9] S. H. Song and K.B. Letaief,"Prior Zero-Forcing for Relaying Primary Signals in Cognitive Network," IEEE Global Telecommunication Conference (GLOBECOM), December 2010.

[10] N. D. Sidropoulos, T. N. Davidson, ans Z.-Q. Luo, "Transmit Beamforming for Physical-Layer Multicasting," IEEE Transactions on Signal Processing, vol. 54, no. 6, pp. 2239-2251, June 2006.

[11] N. Jindal and Z.-Q. Luo, "Capacity Limits of Multiple Antenna Multicast," IEEE International Symposium on Information Theory (ISIT), pp. 1841 - 1845, June 2006.

[12] C. Masouros and E. Alsusa,"Dynamic Linear Precoding for the exploitation of Known Interference in MIMO Broadcast Systems," IEEE Transactions On Communications, vol. 8, no. 3, pp. 1396 - 1404, March 2009.

[13] C. Masouros, "Correlation Rotation Linear Precoding for MIMO Broadcast Communications," IEEE Transaction on Signal Processing, vol. 59, no. 1, pp. 252 -262, January 2011.

[14] M. Alodeh, S. Chatzinotas and B. Ottersten, "Data Aware User Selection in the Cognitive Downlink MISO Precoding Systems," invited paper to IEEE International Symposium on Signal Processing and Information Technology (ISSPIT), December 2013.

[15] M. Alodeh, S. Chatzinotas and B. Ottersten, "A Multicast Approach for Constructive Interference Precoding in MISO Downlink Channel," in the proceedings of International Symposium in Information theory (ISIT) 2014, Available on arXiv:1401.6580v2 [cs.IT].

[16] M. Alodeh, S. Chatzinotas and B. Ottersten, "Constructive Multiuser Interference in Symbol Level Precoding for the MISO Downlink Channel," IEEE Transactions on Signal processing 2015, Available on arXiv:1408.4700 [cs.IT].

[17] M. Alodeh, S. Chatzinotas, and B. Ottersten, "Symbol Based Precoding in the Downlink of Cognitive MISO Channel," Crowncom, 2015.

[18] M. Alodeh, S. Chatzinotas and B. Ottersten, "Energy Efficient Symbol-Level Precoding in Multiuser MISO Channels," 16th IEEE Int. Workshop on Signal Process. Adv. in Wireless Communications (SPAWC), June 2015.

[19] M. Alodeh, S. Chatzinotas and B. Ottersten, "Energy Efficient Symbol-Level Precoding in Multiuser MISO Channels Based on Relaxed Detection Region," IEEE Transactions Wireless Communications, vol. 15 , no. 5, pp. 3755 - 3767, May 2016.

[20] M. Alodeh, S. Chatzinotas and B. Ottersten, "Constructive Interference through Symbol Level Precoding for Multi-level Modulation," IEEE Global conference on Communications, December 2015, available on Arxiv arXiv:1504.06750 [cs.IT].

[21] D. Spano, M. Alodeh, S. Chatzinotas, and B. Ottersten, "Per-antenna Power Minimization in Symbol Level Precoding," submitted to IEEE Global Conference on Communications (GLOBECOM), Washington DC, 2016.

[22] A. Kalantari, M. Soltanalian, S. Maleki, S. Chatzinotas and B. Ottersten, "Secure M-PSK Communication Via Directional Modulation," IEEE International Conference on Acoustic, Speech, and Signal Processing, Shanghai-China, March 2016.

[23] A. Kalantari, M. Soltanalian, S. Maleki, S. Chatzinotas and B. Ottersten, "Directional Modulation via Symbol-Level Precoding: A Way to Enhance Security," submitted to IEEE Journal Selected Topics in Signal Processing, January 2016 .
[24] D. Spano, D. Christopoulos, S. Andrenacci, S. Chatzinotas, J. Krause, and B. Ottersten, "Total degradation analysis of precoded signals onto non-linear satellite channels, 21st Ka and Broadband Communications Conference, Oct 2015.

[25] M. Bavad, and S. Blostien, " Minimum probability of error multiuser transmit beamforming," Asilomar Conference on Signals, Systems and Computers, Pacific Grove-CA, November 2015.

[26] H. Boche, M. Schubert, "Resource allocation in multiantenna systems-achieving max-min fairness by optimizing a sum of inverse SIR," IEEE Transactions on Signal Processing, vol. 54 no. 6, pp. 1990-1997, 2006.

[27] M. Bengtsson and B. Ottersten,"Optimal and Suboptimal Transmit beamforming," in Handbook of Antennas in Wireless Communications, L. C. Godara, Ed. CRC Press, 2001.

[28] M. Schubert and H. Boche, "Solution of the Multiuser Downlink Beamforming Problem with Individual SINR Constraints," IEEE Transaction on Vehicular Technology, vol. 53, pp. 1828, January 2004.

[29] S. Boyd and L. Vandenberghe, Convex Optimization, Cambridge University Press. 